\title{
The FDA Mandate to Reassess Benzodiazepines: Alprazolam Induces a Positive Conditioned Place-Preference in Male Rats
}

\author{
Cheyenne Moffett ${ }^{1}$, Katherine J. Kost ${ }^{1}$, Austen Thompson ${ }^{1}$, Michael H. Ossipov ${ }^{1}$, \\ Joseph V. Pergolizzi Jr.,2,3,4, Sumiyo Umeda-Raffa', Robert B. Raffa ${ }^{3,4,6,7}$, \\ Tally M. Largent-Milnes ${ }^{1}$, Todd Vanderah ${ }^{1 *}$
}

\author{
${ }^{1}$ Department of Pharmacology, and Comprehensive Pain and Addiction Center, University of Arizona, Tucson, AZ, USA \\ ${ }^{2}$ NEMA Inc., Naples, FL, USA \\ ${ }^{3}$ Neumentum Inc., Morristown, NJ, USA \\ ${ }^{4}$ Enalare Therapeutics, Naples, FL, USA \\ ${ }^{5}$ Pharmaceutical Sciences (Form. Faculty), Hokkaido University of Science, Hokkaido, Japan \\ ${ }^{6}$ Temple University School of Pharmacy (Prof Em.), Philadelphia, PA, USA \\ ${ }^{7}$ College of Pharmacy (Adjunct), University of Arizona, Tucson, AZ, USA \\ Email: *vanderah@arizona.edu
}

How to cite this paper: Moffett, C., Kost, K.J., Thompson, A., Ossipov, M.H., Pergolizzi Jr., J.V., Umeda-Raffa, S., Raffa, R.B., Largent-Milnes, T.M. and Vanderah, T. (2021) The FDA Mandate to Reassess Benzodiazepines: Alprazolam Induces a Positive Conditioned Place-Preference in Male Rats. Journal of Biosciences and Medicines, 9, 1-8.

https://doi.org/10.4236/jbm.2021.93001

Received: January 30, 2021

Accepted: March 8, 2021

Published: March 11, 2021

Copyright $\odot 2021$ by author(s) and Scientific Research Publishing Inc. This work is licensed under the Creative Commons Attribution International License (CC BY 4.0).

http://creativecommons.org/licenses/by/4.0/ (c) (i) Open Access

\begin{abstract}
On September 23, 2020, in order "To address the serious risks of abuse, addiction, physical dependence, and withdrawal reactions, the U.S. Food and Drug Administration (FDA) is requiring the Boxed Warning be updated for all benzodiazepine medicines". With this announcement, the FDA proclaimed that much more needs to be known about the initiation, continuation, and discontinuation of these widely-used drugs. Unfortunately, relevant information is lacking, since for many years, there has been a notable sparsity in the funding and conduct of basic and clinical research on these drugs. In order to begin to fill the void, it is valuable to (re)examine animal models. We here describe a model of conditioned place-preference (CPP) for rats and for the first time, to our knowledge, show that the representative benzodiazepine alprazolam induces positive place-preference in male rats.
\end{abstract}

\section{Keywords}

Benzodiazepines, Conditioned Place-Preference, Alprazolam, Reinforcement, Affective State

\section{Introduction}

The first benzodiazepines (BZDs) (Figure 1) were introduced into medical 


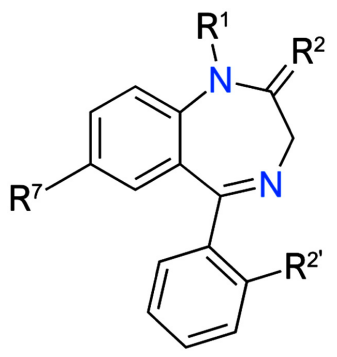

Figure 1. Benzodiazepines are a chemical family of compounds comprised of the fusion of a 6-carbon benzene ring and a 7-carbon diazepine ring. Substitutions at sites indicated by $\mathrm{R}$ result in the large number and variety of analogs.

practice with the development of chlordiazepoxide in 1960 and diazepam in 1962. Due to apparent superior tolerability and safety compared to extant drug therapy, the BZDs were rapidly accepted by the medical community and patients, and widely supplanted other drugs (e.g., the barbiturates) [1]. There are currently more than a dozen different BZDs approved by the United States Food and Drug Administration (FDA) for several indications, including anxiety, insomnia, muscle relaxation, spasticity due to central nervous system pathology, and epilepsy, as well as for intra-operative use (for their amnesic and antianxiety properties) [2]. They have varying degrees of potency, speed of onset, metabolism, and duration of action. An advantage over the barbiturates is that the benzodiazepines cause significantly less respiratory depression, and overdose with benzodiazepines alone is rarely lethal [3]. In part because of their favorable safety profile and the perception that they have little abuse and dependence liability, prescribing of BZDs increased rapidly throughout the 1970s and they became among the most commonly prescribed drugs, with use increasing with advancing age (Figure 2) [4]. In the United States, the number of ambulatory visits with one or more prescriptions for a BZD was 62.6 million in 2015 [5], with alprazolam, clonazepam, diazepam, and lorazepam being the four most-widely prescribed [6].

With the increased use of BZDs came an increasing awareness that they may not be as free from negative consequences as initially believed. In fact, as early as the 1980s, evidence of potential abuse and dependence was accumulating [4]. Although they are associated with a low risk of mortality when used alone, the BZDs interact synergistically with opioids, ethanol, and other central nervous system depressants (likely involving the multiplicative contribution of $\mathrm{GABA}_{\mathrm{A}}$ receptor-related medullary suppression, relaxation of intercostal skeletal muscles, and reduced response to $\mathrm{CO}_{2}$ ) resulting in an increased potential for fatal overdoses. In fact, most instances of fatal BZD overdose include concomitant use of alcohol or opioids. Tolerance to the different effects of BZDs develops at variable rates, with tolerance to the hypnotic effects occurring more rapidly than tolerance to the anxiolytic effects, to the point that BZDs may lose their efficacy 


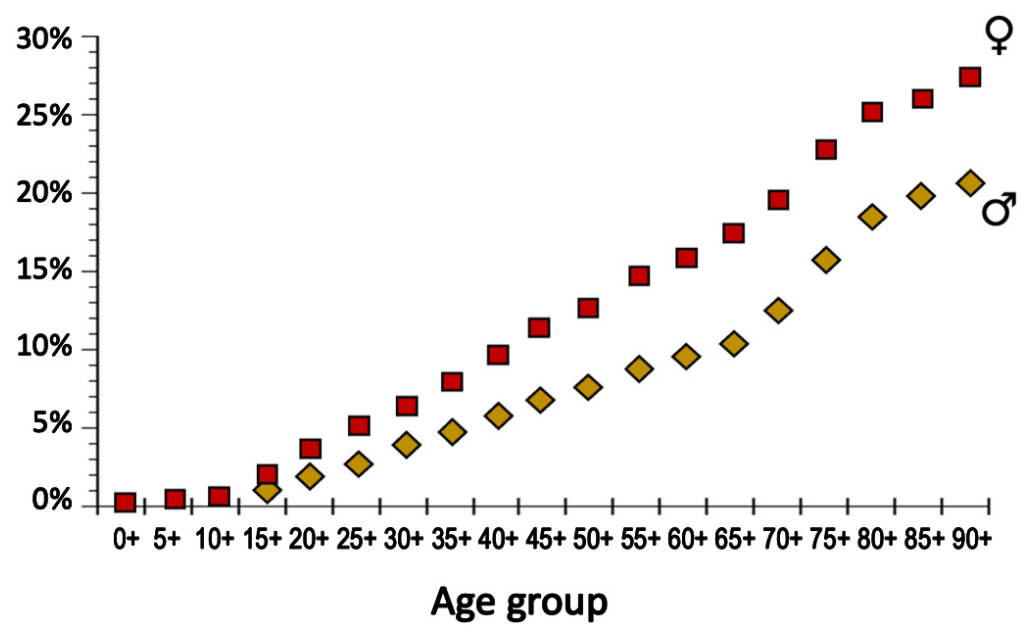

Figure 2. Increase in benzodiazepine prescription rates as a function of age. https://www.ti.ubc.ca/2004/12/31/use-of-benzodiazepines-in-bc-is-it-consisten t-with-recommendations/.

after continued use [3]. The use of BZDs also results in memory impairment and difficulty coordinating movements, resulting in increased risk of falls, particularly in the older population [6]. In addition, there have been numerous reports about the difficulty of long-term withdrawal symptoms after discontinuing the use of benzodiazepines. The most common withdrawal symptom from doses within the therapeutic range includes anxiety, autonomic instability, insomnia, and sensory hypersensitivity [3]. Abrupt withdrawal from BZDs after long-term use can lead to life-threatening seizures, delirium, and death [6].

Drugs associated with abuse potential act in part by activating reward centers of the brain [7]. In this context, reward refers to a positive, or pleasant, affective experience ("liking") [8]. An animal model used for the assessment of rewarding stimuli is conditioned-place-preference (CPP). In CPP, an animal learns to associate one of a pair of environmental cues (locale) with the rewarding stimulus. During evaluation, an animal will spend a significantly greater amount of time in the locale ("paired" chamber in this case) in which it was administered the rewarding drug than it will in a locale in which it was not administered the drug ("neutral" chamber). "Disliking" can also be measured: conditioned place-aversion (CPA) is demonstrated when the animal spends less time in the "paired" chamber. In the present study, we show that the most popular BZD, alprazolam, induces positive place-preference in male rats.

\section{Methods}

Male Sprague Dawley rats obtained from Envigo Laboratories (Indianapolis, IN) weighing 250 - $350 \mathrm{~g}$ were maintained in a climate-controlled room on a $12 \mathrm{hr}$ light/dark cycle. Food and water were available ad libitum. The experiments were performed in accordance with the policies and recommendations of the International Association for the Study of Pain (IASP) and the National Institutes of Health (NIH) guidelines for the handling and use of laboratory animals. 
The study was approved by the Institutional Animal Care and Use Committee (IACUC) of the University of Arizona. All efforts were made to minimize animal suffering and reduce the number of rats used. The experiments were conducted by an experimenter blinded to treatment groups.

The CPP protocol was conducted over a 14-day period using a test system (San Diego Instruments) consisting of two "pairing" chambers and a smaller, well-lit "middle" chamber. The pairing chambers were dimly lit, and consisted of solid colored walls (grey) with a textured floor, and the second chamber was markedly different: it had striped walls (black and white) and a smooth floor. The smaller middle chamber was used to introduce the animal to the system, and had no artificial odor associated with it. On the first day, a rat was individually placed in the middle chamber, and had free access to all chambers over a period of 15 minutes. Automated infrared beams were used to quantify the time spent in each of the chambers (San Diego Instruments; San Diego, CA). During this period, the time spent in each chamber was recorded to ensure that there were no inherent biases for one chamber over another. If an animal showed an innate bias towards one chamber (defined as spending more than $80 \%$ of the time there), it was removed from the study without further evaluation. On the second day of testing, the animals entered the conditioning protocol. They were injected with alprazolam ( $3 \mathrm{mg} / \mathrm{kg}$, s.c.; $\mathrm{N}=14$ ) or vehicle ( $10 \% \mathrm{DMSO}, 10 \%$ Tween-80, and $80 \%$ saline, $1 \mathrm{~mL} / \mathrm{kg} ; \mathrm{N}=15$ ) and were immediately placed into one of the two pairing chambers for $30 \mathrm{~min}$, without access to the other chambers. This was the chamber in which the animals would receive drug injection, so was designated the "paired chamber"; the chamber not associated with drug injection was designated the "neutral chamber". The pairing for each animal was balanced, i.e., half of the rats received drug (or vehicle) treatment in the solid-colored chamber, and the other half received drug (or vehicle) in the striped chamber. This pairing was repeated for a total of 5 drug exposures.

Testing was performed on the following day (day 5 of the protocol), by placing the animals in the middle chamber and allowing them access to roam freely across all chambers. Test day did not include any injections. The time spent in each chamber was recorded, and a difference score was derived by subtracting the time spent in the neutral chamber from the time spent in the paired chamber. In this paradigm a significant positive difference score ( $>50$ s over baseline) is indicative of preference for the drug-pairing (CPP); a significant negative difference score (>50 s decrease from baseline) is indicative of aversion to the drug-pairing CPA.

The mean time that the rats spent in the paired-chamber and the neutral-chamber was calculated for each group $( \pm S E M)$. Student's t-test was used to evaluate differences between the mean times for statistical significance $(\mathrm{P}>$ $0.05)$.

\section{Results}

The rats that received vehicle injection did not display significant difference in 
mean time spent in the paired chamber between baseline and test day. That is, they did not develop a preference for the vehicle-paired chamber compared to the other (nonpaired) chamber. In contrast, the animals that were administered alprazolam ( $3 \mathrm{mg} / \mathrm{kg}$, s.c., $\mathrm{N}=14)$ on the conditioning day showed a statistically significant $(\mathrm{p} \leq 0.01)$ increase in time spent in the drug-paired chamber compared to baseline (Figure 3). Figure 4 shows the scatter of the time spent in the paired chamber for each individual rat at baseline and on test day. The data appear to be evenly distributed about the mean.

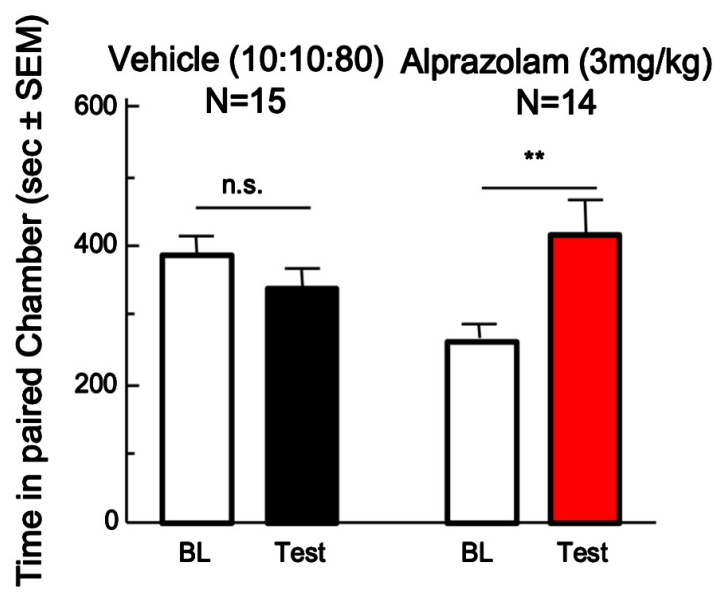

Figure 3. The mean ( \pm SEM) time, in seconds, spent in the paired chamber at baseline and on test day for rats receiving vehicle or alprazolam (3 $\mathrm{mg} / \mathrm{kg}$, s.c.) is shown. There was so significant difference in time spent in the paired chamber between baseline and test day for animals who were injected with the vehicle. In contrast, alprazolam injection resulted in a significant $(\mathrm{p} \leq 0.01)$ increase in time spent in the paired chamber after treatment. n.s. means non-significant. ${ }^{*}=\mathrm{P}<0.01$.

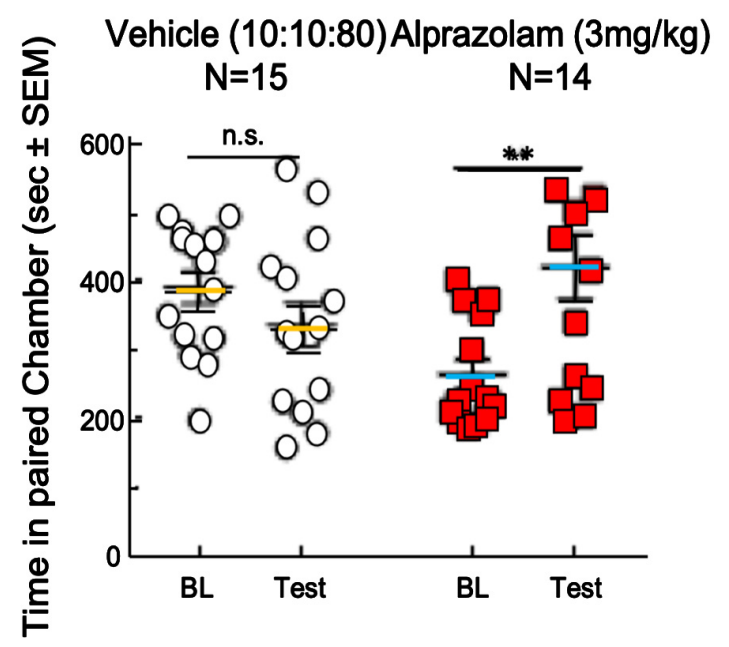

Figure 4. Data for each treatment group at baseline and on test day appear to be evenly scattered about the mean. n.s. means non-significant. ${ }^{*}=\mathrm{P}<0.01$. 


\section{Vehicle (10:10:80) $\mathrm{N}=15$}

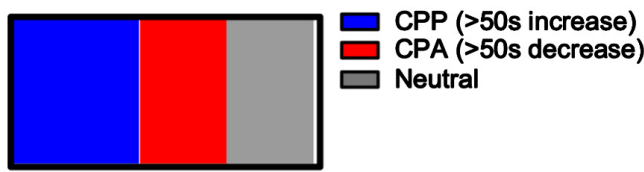

\section{Alprazolam $(3 \mathrm{mg} / \mathrm{kg})$ $\mathrm{N}=14$}

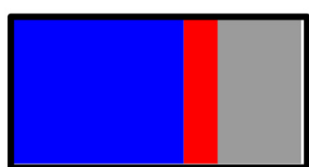

CPP (>50s increase) $\square$ CPA (>50s decrease) $\square$ Neutral

Figure 5. Data for the reward liability of vehicle or alprazolam. Vehicle-induced CPP (40\%), Neutral (33\%), or CPA (27\%) behaviors after repeated exposure. Alprazolam-induced CPP (60\%), Neutral $(28 \%)$, or CPA $(12 \%)$ outcomes in male rats.

Among the rats that received the vehicle injection on conditioning day, an approximately equal number of rats showed a preference (indicated by an increase in time spent in the paired chamber of $>50 \mathrm{sec}$ ), an aversion (indicated by a decrease of $>50 \mathrm{sec}$ in time spent in the paired chamber), or no preference (the time spent in either chamber differed by $\leq 50 \mathrm{sec}$ ) (Figure 5). Among the animals treated with alprazolam, there was a greater proportion that showed a preference compared to those who showed aversion or no preference (Figure 5).

\section{Discussion}

Several previous preclinical studies have investigated benzodiazepines' potential influence on the rewarding properties of abused drugs including the opioid heroin and the central nervous system stimulant methamphetamine [9] [10]. For example, alprazolam at doses of $0.125 \mathrm{mg} / \mathrm{kg}$ to $0.5 \mathrm{mg} / \mathrm{kg}$ given with heroin demonstrated an interaction that results in a reliable conditioned place preference [9] [10] [11]. Oxazepam significantly reduced methamphetamine self-administration, whereas alprazolam significantly enhanced methamphetamine self-administration [12]. Furthermore, a preclinical study was performed to determine whether benzodiazepines could inhibit the reinstatement of cocaine seeking behavior. Pretreatment of rats with alprazolam ( 2 or $4 \mathrm{mg} / \mathrm{kg}$, ip) or oxazepam (20 or $40 \mathrm{mg} / \mathrm{kg}$, ip) reversed the conditioned reinforcer-induced reinstatement of extinguished cocaine-seeking behavior at doses that did not reliably affect the conditioned reinforcer-induced reinstatement of extinguished food seeking, suggesting that benzodiazepines may be useful in blocking the ability of environmental cues to stimulate cocaine seeking [13].

Surprisingly, and despite growing evidence of potential abuse and dependence of benzodiazepines in humans [4], very few studies have investigated benzodiazepines for their ability to produce rewarding behavior by themselves. Matsuza- 
wa et al. (in 2000) demonstrated that the benzodiazepine, diazepam, did not result in a positive condition placed preference in rats that were exposed to stress [14]. Here we demonstrate that alprazolam when given once a day at the dose of $3 \mathrm{mg} / \mathrm{kg}$ over 5 days to male rats can result in a positive conditioned place preference when compared to the animal's baseline and to vehicle. These data suggest that alprazolam in male rats induces rewarding-like behavior and have the potential to result in addiction.

Unfortunately, the paucity of studies on the mechanism of benzodiazepines' rewarding activity in animals and/or humans falls short from understanding how benzodiazepines may regulate dopamine release and/or activation of reward pathways. The preclinical study by Spence et al. (in 2016) demonstrated that pretreatment with an antagonist for the benzodiaz-epine-binding site on the $\mathrm{GABA}_{\mathrm{A}}$ receptor (i.e., flumazenil) completely blocked the effects of alprazolam on methamphetamine self-administration by rats [12]. Both flumazenil and a TSPO (Translocator protein) antagonist (viz., PK11195) partially reversed the effects of oxazepam on methamphetamine self-administration. However, when these two antagonists were combined, the effects of oxazepam were completely reversed, suggesting that both $\mathrm{GABA}_{\mathrm{A}}$ and TSPO may be responsible for benzodiazepine's role in methamphetamine rewarding activities. A recent clinical study using perfusion magnetic resonance imaging demonstrated that alprazolam acutely increases perfusion in the nucleus accumbens, a key reward-processing region of the central nervous system [15].

\section{Funding}

The study was funded by an unrestricted grant from The Alliance for Benzodiazepine Best Practices: https://benzoreform.org/.

\section{Conflicts of Interest}

The authors declare no conflicts of interest regarding the publication of this paper.

\section{References}

[1] Schmitz, A. (2016) Benzodiazepine Use, Misuse, and Abuse: A Review. Mental Health Clinician, 6, 120-126. https://doi.org/10.9740/mhc.2016.05.120

[2] Griffin, C.E., Kaye, A.M., Bueno, F.R. and Kaye, A.D. (2013) Benzodiazepine Pharmacology and Central Nervous System-Mediated Effects. Ochsner Journal, 13, 214-223.

[3] Longo, L.P. and Johnson, B. (2000) Addiction: Part I. Benzodiazepines-Side Effects, Abuse Risk and Alternatives. American Family Physician, 61, 2121-2128.

[4] Wick, J.Y. (2013) The History of Benzodiazepines. The Consultant Pharmacist, 28, 538-548. https://doi.org/10.4140/TCP.n.2013.538

[5] Agarwal, S.D. and Landon, B.E. (2019) Patterns in Outpatient Benzodiazepine Prescribing in the United States. JAMA Network Open, 2, e187399.

https://doi.org/10.1001/jamanetworkopen.2018.7399 
[6] Fluyau, D., Revadigar, N. and Manobianco, B.E. (2018) Challenges of the Pharmacological Management of Benzodiazepine Withdrawal, Dependence, and Discontinuation. Therapeutic Advances in Psychopharmacology, 8, 147-168. https://doi.org/10.1177/2045125317753340

[7] Volkow, N.D., Wang, G.-J., Fowler, J.S., Tomasi, D., Telang, F. and Baler, R. (2010) Addiction: Decreased Reward Sensitivity and Increased Expectation Sensitivity Conspire to Overwhelm the Brain's Control Circuit. BioEssays, 32, 748-755. https://doi.org/10.1002/bies.201000042

[8] White, N.M. (2011) Chapter 3 Reward: What Is It? How Can It Be Inferred from Behavior? In: Gottfried, J., Ed., Neurobiology of Sensation and Reward, CRC Press/Taylor \& Francis, Boca Raton, 45-60.

[9] Walker, B.M. and Ettenberg, A. (2003) The Effects of Alprazolam on Conditioned Place Preferences Produced by Intravenous Heroin. Pharmacology Biochemistry and Behavior, 75, 75-80. https://doi.org/10.1016/S0091-3057(03)00043-1

[10] Walker, B.M. and Ettenberg, A. (2005) Intra-Ventral Tegmental Area Heroin-Induced Place Preferences in Rats Are Potentiated by Peripherally Administered Alprazolam. Pharmacology Biochemistry and Behavior, 82, 470-477. https://doi.org/10.1016/j.pbb.2005.10.002

[11] Tzschentke, T.M. (2007) REVIEW ON CPP: Measuring Reward with the Conditioned Place Preference (CPP) Paradigm: Update of the Last Decade. Addiction Biology, 12, 227-462. https://doi.org/10.1111/j.1369-1600.2007.00070.x

[12] Spence, A.L., Guerin, G.F. and Goeders, N.E. (2016) The Differential Effects of Alprazolam and Oxazepam on Methamphetamine Self-Administration in Rats. Drug and Alcohol Dependence, 166, 209-217. https://doi.org/10.1016/j.drugalcdep.2016.07.015

[13] Goeders, N.E., Clampitt, D.M., Keller, C., Sharma, M. and Guerin, G.F. (2009) Alprazolam and Oxazepam Block the Cue-Induced Reinstatement of Extinguished Cocaine Seeking in Rats. Psychopharmacology, 201, 581-588. https://doi.org/10.1007/s00213-008-1326-1

[14] Matsuzawa, S., Suzuki, T. and Misawa, M. (2000) Ethanol, But Not the Anxiolytic Drugs Buspirone and Diazepam, Produces a Conditioned Place Preference in Rats Exposed to Conditioned Fear Stress. Pharmacology Biochemistry and Behavior, 65, 281-288. https://doi.org/10.1016/S0091-3057(99)00224-5

[15] Wolf, D.H., Pinkham, A.E., Satterthwaite, T.D., Ruparel, K., Elliott, M.A., Valdez, J., et al. (2013) Oral Alprazolam Acutely Increases Nucleus Accumbens Perfusion. Molecular Psychiatry, 18, 960-961. https://doi.org/10.1038/mp.2012.139 